\title{
On Stochastic Evolutions and Superconformal Field Theory
}

\author{
Jasbir Nagi* and Jørgen Rasmussen ${ }^{\dagger}$ \\ *DAMTP, University of Cambridge, Wilberforce Road \\ Cambridge, UK, CB3 OWA \\ † Centre de recherches mathématiques, Université de Montréal \\ Case postale 6128, succursale centre-ville, Montréal, Qc, Canada H3C 3J7 \\ j.s.nagi@damtp.cam.ac.uk, rasmusse@crm.umontreal.ca
}

\begin{abstract}
Links between certain stochastic evolutions of conformal maps and conformal field theory have been studied in the realm of SLE and by utilizing singular vectors in highestweight modules of the Virasoro algebra. It was recently found that this scenario could be extended to stochastic evolutions of superconformal maps of $N=1$ superspace with links to superconformal field theory and singular vectors of the $N=1$ superconformal algebra in the Neveu-Schwarz sector. Here we discuss the analogous extension to the Ramond sector. We also discuss how the links are modified when an unconventional superconformal structure or superderivative is employed.
\end{abstract}

Keywords: Stochastic evolutions, superconformal field theory, superspace. 


\section{Introduction}

Certain two-dimensional systems at criticality may be described in terms of stochastic Löwner evolutions (SLEs). This was discovered by Schramm [1] and has been developed further in 2, 3, for example. The method involves the study of stochastic evolutions of conformal maps. A particular link to conformal field theory (CFT) has been examined by Bauer and Bernard [4] (see also [5]) and extended in [6]. In these studies, the SLE differential equation is associated to a random walk on the Virasoro group. Based on the representation theory of CFT, a more direct relationship may be established which involves entities conserved in mean under the stochastic process. A key ingredient is the existence of singular vectors in the associated Virasoro highest-weight modules.

This program was extended in [7] to a link between stochastic evolutions of superconformal maps in $N=1$ superspace and the Neveu-Schwarz (NS) sector in $N=1$ superconformal field theory (SCFT). This extension relies on an Ito calculus, not just for commuting and anti-commuting variables as discussed in [8] and references therein, but for general non-commuting objects (e.g. group- or algebra-valued entities) on superspace. The generalization is straightforward, though, and discussed in [7].

Our main objective here is to work out the analogous results in the Ramond sector of $N=1 \mathrm{SCFT}$. This can be done by considering the superconformal condition in the NS sector, but with the odd co-ordinate on superspace having anti-periodic boundary conditions. Alternatively, as was first noticed in [9], and developed further in [10], one can obtain the Ramond sector by considering a different superconformal condition, in which case the odd co-ordinate has periodic boundary conditions. The NS sector is then characterized by anti-periodic boundary conditions on the odd co-ordinate. In both approaches, we shall use the representation theory of the $N=1$ superconformal algebra in the Ramond sector to link stochastic evolutions in $N=1$ superspace to $N=1$ SCFT. For completeness, we also address the similar link based on the alternative superconformal condition in the NS sector, and review some of the original results of [7].

\section{SLE and CFT}

SLE [1, 2, 3], or chordal SLE, describes the evolution of boundaries of simply-connected domains in the complex plane. This may be thought of as Brownian motion on the set of conformal maps $\left\{g_{t}\right\}$ satisfying the Löwner equation

$$
\partial_{t} g_{t}(z)=\frac{2}{g_{t}(z)-\sqrt{\kappa} B_{t}}, \quad g_{0}(z)=z
$$

$B_{t}$ is one-dimensional Brownian motion with $B_{0}=0, \kappa \geq 0$, and the system is often denoted $\mathrm{SLE}_{\kappa}$.

The link to CFT [4] is established by considering Ito differentials of Virasoro group 
elements $\mathcal{G}_{t}$ :

$$
\mathcal{G}_{t}^{-1} d \mathcal{G}_{t}=\left(-2 L_{-2}+\frac{\kappa}{2} L_{-1}^{2}\right) d t+\sqrt{\kappa} L_{-1} d B_{t}, \quad \mathcal{G}_{0}=1
$$

Such group elements are obtained by exponentiating generators of the Virasoro algebra

$$
\left[L_{n}, L_{m}\right]=(n-m) L_{n+m}+\frac{c}{12} n\left(n^{2}-1\right) \delta_{n+m, 0}
$$

where $c$ is the central charge. The conformal transformation generated by a Virasoro group element has a simple action on a primary field of weight $\Delta$. For simplicity, we do not distinguish explicitly between boundary and bulk primary fields, nor do we write the anti-holomorphic part. The transformation generated by $\mathcal{G}_{t}$ reads

$$
\mathcal{G}_{t}^{-1} \phi_{\Delta}(z) \mathcal{G}_{t}=\left(\partial_{z} f_{t}(z)\right)^{\Delta} \phi_{\Delta}\left(f_{t}(z)\right)
$$

for some conformal map $f_{t}$. Using that the Virasoro generators act as

$$
\left[L_{n}, \phi_{\Delta}(z)\right]=\left(z^{n+1} \partial_{z}+\Delta(n+1) z^{n}\right) \phi_{\Delta}(z)
$$

one finds that the conformal map $f_{t}$ associated to the random process $\mathcal{G}_{t}$ (2) must be a solution to the stochastic differential equation

$$
d f_{t}(z)=\frac{2}{f_{t}(z)} d t-\sqrt{\kappa} d B_{t}, \quad f_{0}(z)=z
$$

With the definition

$$
f_{t}(z):=g_{t}(z)-\sqrt{\kappa} B_{t}
$$

this is merely a rewriting of the SLE equation (11).

This link does not teach us to which CFT with central charge $c$ a given $\mathrm{SLE}_{\kappa}$ may be associated. A refinement is established by relating the representation theory of the Virasoro algebra to entities conserved in mean under the random process. Observables of the stochastic process (2) are here thought of as functions on the Virasoro group. With $|\Delta\rangle$ denoting the highest-weight vector of weight $\Delta$ in the Verma module $\mathcal{V}_{\Delta}$, we now follow [4] and consider the time evolution of the expectation value of $\mathcal{G}_{t}|\Delta\rangle$ :

$$
\partial_{t} \mathbf{E}\left[\mathcal{G}_{t}|\Delta\rangle\right]=\mathbf{E}\left[\mathcal{G}_{t}\left(-2 L_{-2}+\frac{\kappa}{2} L_{-1}^{2}\right)|\Delta\rangle\right]
$$

The linear combination $-2 L_{-2}+\frac{\kappa}{2} L_{-1}^{2}$ will produce a singular vector when acting on the highest-weight vector provided

$$
c_{\kappa}=1-\frac{3(4-\kappa)^{2}}{2 \kappa}, \quad \Delta_{\kappa}=\frac{6-\kappa}{2 \kappa}
$$

In this case the right-hand side of (8) vanishes in the module obtained by factoring out the singular vector from the reducible Verma module $\mathcal{V}_{\Delta}$. The representation theory of the factor module is thereby linked to the description of entities conserved in mean. 


\section{Superspace and SCFT}

Here we shall give a very brief summary of results from the theory of $N=1$ superspace and $N=1$ SCFT required in the following. We refer to [11, 10] and references therein for recent accounts on the subject. As we only consider $N=1$ superspace and $N=1$ SCFT we shall simply refer to them as superspace and SCFT, respectively.

\subsection{Superconformal maps}

Let

$$
(z, \theta) \mapsto\left(z^{\prime}, \theta^{\prime}\right), \quad\left\{\begin{array}{l}
z^{\prime}=g(z)+\theta \gamma(z) \\
\theta^{\prime}=\tau(z)+\theta s(z)
\end{array}\right.
$$

denote a general superspace co-ordinate transformation. The conventionally associated superderivative reads

$$
D=\partial_{\theta}+\theta \partial_{z}
$$

Here $\theta, \theta^{\prime}, \gamma$ and $\tau$ are anti-commuting or (Grassmann) odd entities, while $z, z^{\prime}, g$ and $s$ are even. A superconformal transformation corresponding to (11) is characterized by

$$
D z^{\prime}=\theta^{\prime} D \theta^{\prime}
$$

in which case we have

$$
\gamma(z)=\tau(z) s(z), \quad \partial_{z} g(z)=s^{2}(z)-\tau(z) \partial_{z} \tau(z)
$$

and

$$
D=\left(D \theta^{\prime}\right) D^{\prime}, \quad D^{\prime}=\partial_{\theta^{\prime}}+\theta^{\prime} \partial_{z^{\prime}}
$$

We shall be interested in locally invertible superconformal maps implying that the complex part of $z^{\prime}$ is non-vanishing if the complex part of $z$ is non-vanishing. We assume this is the case for $z \neq 0$.

The superconformal transformations are generated by $T$ and $G$ with modes $L_{n}$ and $G_{r}$ satisfying the superconformal algebra

$$
\begin{aligned}
{\left[L_{n}, L_{m}\right] } & =(n-m) L_{n+m}+\frac{c}{12} n\left(n^{2}-1\right) \delta_{n+m, 0} \\
{\left[L_{n}, G_{r}\right] } & =\left(\frac{n}{2}-r\right) G_{n+r} \\
\left\{G_{r}, G_{s}\right\} & =2 L_{r+s}+\frac{c}{3}\left(r^{2}-\frac{1}{4}\right) \delta_{r+s, 0}
\end{aligned}
$$

The algebra is said to be in the Neveu-Schwarz (NS) sector when the supercurrent $G$ has half-integer modes, and in the Ramond sector when $G$ has integer modes. Branch cuts from square roots are known to appear in the Ramond sector. 
Below it will be discussed how one can avoid these branch cuts in the Ramond sector by introducing a different superconformal structure. To this end, we consider the superderivative

$$
\mathcal{D}=\partial_{\theta}+\theta z \partial_{z}
$$

It is accompanied by a modified condition for a map (10) to be superconformal:

$$
\mathcal{D} z^{\prime}=\theta^{\prime} z^{\prime} \mathcal{D} \theta^{\prime}
$$

whereas (14) remains valid in the sense that

$$
\mathcal{D}=\left(\mathcal{D} \theta^{\prime}\right) \mathcal{D}^{\prime}, \quad \mathcal{D}^{\prime}=\partial_{\theta^{\prime}}+\theta^{\prime} z^{\prime} \partial_{z^{\prime}}
$$

The generators of these superconformal transformations also satisfy (15). In particular, the modes of the supercurrent are still integer in the Ramond sector and half-integer in the NS sector. Branch cuts now appear in the NS sector instead of the Ramond sector, and we have periodic boundary conditions on the odd co-ordinate in the Ramond sector, and anti-periodic boundary conditions in the NS sector. This is the opposite of the usual characterization of the sectors based on the conventional superconformal structure defined by $D$.

\subsection{Primary fields and singular vectors}

The superconformal transformations generated by Virasoro supergroup elements $\mathcal{G}$ extend the ordinary conformal case (44). Supergroup elements may be constructed by exponentiating the generators of the superconformal algebra. Modes of the supercurrent must in that case be accompanied by Grassmann odd parameters. Primary fields are defined by having simple transformation properties with respect to the superconformal generators (15). With $\Delta$ denoting the (super)conformal dimension, a primary field corresponding to the superderivative (111) transforms as

$$
\mathcal{G}^{-1} \Phi_{\Delta}(z, \theta) \mathcal{G}=\left(D \theta^{\prime}\right)^{2 \Delta} \Phi_{\Delta}\left(z^{\prime}, \theta^{\prime}\right)
$$

whereas it was found in [10] that a primary field corresponding to the alternative superderivative (16) transforms as

$$
\mathcal{G}^{-1} \Phi_{\Delta}(z, \theta) \mathcal{G}=\left(\frac{z^{\prime}}{z}\left(\mathcal{D} \theta^{\prime}\right)^{2}\right)^{\Delta} \Phi_{\Delta}\left(z^{\prime}, \theta^{\prime}\right)
$$

The action of the generators on a primary field thus depends on the superderivative. From (19) we have

$$
\begin{aligned}
& {\left[L_{n}, \Phi_{\Delta}(z, \theta)\right]=\left(z^{n+1} \partial_{z}+\frac{1}{2}(n+1) z^{n} \theta \partial_{\theta}+\Delta(n+1) z^{n}\right) \Phi_{\Delta}(z, \theta)} \\
& {\left[G_{r}, \Phi_{\Delta}(z, \theta)\right]=\left(-\theta z^{r+1 / 2} \partial_{z}+z^{r+1 / 2} \partial_{\theta}-\Delta(2 r+1) \theta z^{r-1 / 2}\right) \Phi_{\Delta}(z, \theta)}
\end{aligned}
$$


with $n \in \mathbb{Z}$ and $r \in \mathbb{Z}+\frac{1}{2}$, while (20) corresponds to

$$
\begin{aligned}
{\left[L_{n}, \Phi_{\Delta}(z, \theta)\right] } & =\left(z^{n+1} \partial_{z}+\frac{n}{2} z^{n} \theta \partial_{\theta}+\Delta(n+1) z^{n}\right) \Phi_{\Delta}(z, \theta) \\
{\left[G_{r}, \Phi_{\Delta}(z, \theta)\right] } & =\left(-\theta z^{r+1} \partial_{z}+z^{r} \partial_{\theta}-\Delta(2 r+1) \theta z^{r}\right) \Phi_{\Delta}(z, \theta)
\end{aligned}
$$

where $n, r \in \mathbb{Z}$. The commutation relations of the operators $L_{n}, G_{r}$ in (22) can be explicitly calculated and hence can be shown to give rise to a representation of the $N=1$ Ramond superconformal algebra. Likewise, the more familiar operators in (21) generate the $N=1 \mathrm{NS}$ superconformal algebra. As indicated, we are considering even primary fields only.

The discussion above assumes periodic boundary conditions on the odd co-ordinate and hence on the supercurrent $G(z)=\sum_{r} G_{r} z^{-r-3 / 2}$, i.e., $G\left(z e^{2 \pi i}\right)=G(z)$. For anti-

periodic boundary conditions where $G\left(z e^{2 \pi i}\right)=-G(z)$, the mode of $G_{r}$ in (21) would be integer (and thus generate the Ramond algebra), while in (22) it would be half-integer (and thus generate the NS algebra).

A highest-weight module of the superconformal algebra is reducible if it contains a submodule generated from a singular vector. In the NS sector, the simplest non-trivial singular vector in a highest-weight module with highest weight $\Delta$ appears at level $3 / 2$ and is given by

$$
|\chi ; 3 / 2\rangle=\left(\left(\Delta+\frac{1}{2}\right) G_{-3 / 2}-L_{-1} G_{-1 / 2}\right)|\Delta\rangle
$$

provided

$$
(2 \Delta+1) c=3 \Delta(3-2 \Delta)
$$

In the Ramond sector, the similar vector appears at level 1:

$$
|\chi ; 1\rangle=\left((8 \Delta+c) L_{-1}-6 G_{-1} G_{0}\right)|\Delta\rangle
$$

provided

$$
(16 \Delta+3) c=8 \Delta(9-16 \Delta)
$$

\section{Graded stochastic evolutions and SCFT}

We first consider the stochastic differential

$$
\mathcal{G}_{t}^{-1} d \mathcal{G}_{t}=\alpha d t+\sum_{i=1}^{b} \beta_{i} d B_{t}^{(i)}, \quad \mathcal{G}_{0}=1
$$

which we can think of as a random walk on the Virasoro supergroup. The coefficients $\alpha$ and $\beta_{i}$ are even and generically non-commutative expressions in the generators of the superconformal algebra. The differential is based on $b$-dimensional Brownian motion, 
$\bar{B}_{t}=\left(B_{t}^{(1)}, \ldots, B_{t}^{(b)}\right)$, with $B_{0}^{(i)}=0$. The associated Ito calculus treats the basic differentials according to the rules

$$
(d t)^{2}=d t d B_{t}^{(i)}=0, \quad d B_{t}^{(i)} d B_{t}^{(j)}=\delta_{i j} d t
$$

The Ito differential of the element inverse to $\mathcal{G}_{t}$ is given by

$$
d\left(\mathcal{G}_{t}^{-1}\right) \mathcal{G}_{t}=\left(-\alpha+\sum_{i=1}^{b} \beta_{i}^{2}\right) d t-\sum_{i=1}^{b} \beta_{i} d B_{t}^{(i)}
$$

The superconformal transformation generated by $\mathcal{G}_{t}$ acts on a primary field as (19) or (201), which means that the new superspace co-ordinate $\left(z_{t}^{\prime}, \theta_{t}^{\prime}\right)$ becomes a stochastic function of $(z, \theta)$, parameterized by $t \in \mathbb{R}_{\geq 0}$. To relax the notation, the subscript $t$ will often be suppressed below. A goal is to compute the Ito differential of both sides of (19) or (20) and thereby relate the stochastic differential equations of $\mathcal{G}_{t}$ and $\left(z^{\prime}, \theta^{\prime}\right)$. In either case, the Ito differential of the left-hand side reads

$$
\begin{aligned}
d\left(\mathcal{G}_{t}^{-1} \Phi_{\Delta} \mathcal{G}_{t}\right)= & d\left(\mathcal{G}_{t}^{-1}\right) \Phi_{\Delta} \mathcal{G}_{t}+\mathcal{G}_{t}^{-1} \Phi_{\Delta} d \mathcal{G}_{t}+d\left(\mathcal{G}_{t}^{-1}\right) \Phi_{\Delta} d \mathcal{G}_{t} \\
= & \left(-\left[\alpha_{0}, \mathcal{G}_{t}^{-1} \Phi_{\Delta} \mathcal{G}_{t}\right]+\frac{1}{2} \sum_{i=1}^{b}\left[\beta_{i},\left[\beta_{i}, \mathcal{G}_{t}^{-1} \Phi_{\Delta} \mathcal{G}_{t}\right]\right]\right) d t \\
& -\sum_{i=1}^{b}\left[\beta_{i}, \mathcal{G}_{t}^{-1} \Phi_{\Delta} \mathcal{G}_{t}\right] d B_{t}^{(i)}
\end{aligned}
$$

where we have introduced $\alpha_{0}=\alpha-\frac{1}{2} \sum_{i=1}^{b} \beta_{i}^{2}$. A direct comparison of (301) with the Ito differential of the right-hand side of (19) or (20) requires that $\beta_{i}$ and $\alpha_{0}$ are linear in the generators:

$$
\begin{aligned}
\alpha_{0} & =\sum_{n} y_{0, n} L_{n}+\sum_{r} \eta_{0, r} G_{r} \\
\beta_{i} & =\sum_{n} y_{i, n} L_{n}+\sum_{r} \eta_{i, r} G_{r}
\end{aligned}
$$

Here $n$ is integer while $r$ is integer or half-integer depending on the sector.

With the subscript $t$ suppressed, we can express the Ito differentials of the superspace co-ordinates as

$$
\begin{array}{rlrl}
d z^{\prime} & =z_{0}^{\prime} d t+\sum_{i=1}^{b} z_{i}^{\prime} d B_{t}^{(i)}, & \left.z^{\prime}\right|_{t=0}=z \\
d \theta^{\prime}=\theta_{0}^{\prime} d t+\sum_{i=1}^{b} \theta_{i}^{\prime} d B_{t}^{(i)}, & \left.\theta^{\prime}\right|_{t=0}=\theta
\end{array}
$$

needed when computing the Ito differential of the right-hand sides of (19) and (20). The initial conditions are required to match the initial condition on $\mathcal{G}$ in (27). 


\subsection{General link for conventional superderivative $D$}

The computation of the Ito differential of the right-hand side of (19), corresponding to the conventional superderivative $D$, was carried out in [7]. The result for the NS sector was subsequently compared to (30). The calculation for the Ramond sector, where $r$ is integer rather than half-integer, follows in exactly the same way. One uses the same definition of primary field (21) and require that it be single valued. The same formulae are obtained, except replacing $r \in \mathbb{Z}+\frac{1}{2}$ with $r \in \mathbb{Z}$. Without specifying this summation range explicitly, the solutions in the two sectors may both be written as ${ }^{1}$

$$
\begin{aligned}
z_{i}^{\prime} & =-\sum_{n \in \mathbb{Z}} y_{i, n}\left(z^{\prime}\right)^{n+1}+\sum_{r} \eta_{i, r} \theta^{\prime}\left(z^{\prime}\right)^{r+\frac{1}{2}} \\
\theta_{i}^{\prime} & =-\frac{1}{2} \sum_{n \in \mathbb{Z}}(n+1) \theta^{\prime} y_{i, n}\left(z^{\prime}\right)^{n}-\sum_{r} \eta_{i, r}\left(z^{\prime}\right)^{r+\frac{1}{2}}
\end{aligned}
$$

and

$$
\begin{aligned}
& z_{0}^{\prime}=-\sum_{n \in \mathbb{Z}} y_{0, n}\left(z^{\prime}\right)^{n+1}+\sum_{r} \eta_{0, r} \theta^{\prime}\left(z^{\prime}\right)^{r+\frac{1}{2}}+\frac{1}{2} \sum_{i=1}^{b}\left(z_{i}^{\prime} \partial_{z^{\prime}}+\theta_{i}^{\prime} \partial_{\theta^{\prime}}\right) z_{i}^{\prime} \\
& \theta_{0}^{\prime}=-\frac{1}{2} \sum_{n \in \mathbb{Z}}(n+1) \theta^{\prime} y_{0, n}\left(z^{\prime}\right)^{n}-\sum_{r} \eta_{0, r}\left(z^{\prime}\right)^{r+\frac{1}{2}}+\frac{1}{2} \sum_{i=1}^{b}\left(z_{i}^{\prime} \partial_{z^{\prime}}+\theta_{i}^{\prime} \partial_{\theta^{\prime}}\right) \theta_{i}^{\prime}
\end{aligned}
$$

Using (33), one may of course eliminate $z_{i}^{\prime}$ and $\theta_{i}^{\prime}$ from these latter expressions, but the result is then less compact than (34). Only finitely many of the expansion coefficients $y$ and $\eta$ will be chosen non-vanishing rendering the sums in the solution (33) and (34) finite.

In conclusion, the construction above establishes a general link between a class of stochastic evolutions in superspace and SCFT. The stochastic differentials (32) describing the evolution of the superconformal maps are expressed in terms of the parameters of the random walk on the Virasoro supergroup (27), and this has been achieved via the definition of primary fields in SCFT (19). The solution to the stochastic differential equations in the NS sector is given in (33) and (34) when the sum over $r$ is over $r \in \mathbb{Z}+\frac{1}{2}$. The solution in the Ramond sector, where the odd co-ordinate now has anti-periodic boundary conditions, is obtained from the NS solution by replacing the summation over $r \in \mathbb{Z}+\frac{1}{2}$ with $r \in \mathbb{Z}$. As can be seen, this introduces square roots of $z^{\prime}$, and hence a branch cut in the Ramond solution.

\footnotetext{
${ }^{1}$ Here and in the following, brackets may be used to write powers of $z^{\prime}$ as $\left(z^{\prime}\right)^{a}$ instead of $z^{\prime a}$, for example. The parameters preceding these expressions, such as in $y_{i, n}\left(z^{\prime}\right)^{n+1}$, should thus not be confused with functions of $z^{\prime}$.
} 


\subsection{General link for alternative superderivative $\mathcal{D}$}

Rather than using (19) and the conventional superderivative $D$, one could try using (20) and the alternative superderivative $\mathcal{D}$. As we shall see, the branch cuts in the Ramond sector encountered above are thereby avoided but reappear in the NS sector instead.

To this end, we now consider the case where $\left(z^{\prime}, \theta^{\prime}\right)$ and $\mathcal{G}$ in (20) evolve as stochastic processes, again parameterized by $t \in \mathbb{R}_{\geq 0}$. The Ito differential of the left-hand side of (20) is given by (30), while the Ito differential of the right-hand side is found to be

$$
\begin{aligned}
& d\left(\left(\frac{z^{\prime}}{z}\right)^{\Delta}\left(\mathcal{D} \theta^{\prime}\right)^{2 \Delta} \Phi\left(z^{\prime}, \theta^{\prime}\right)\right) \\
= & \frac{\left(\mathcal{D} \theta^{\prime}\right)^{2 \Delta}}{z^{\Delta}}\left(d z^{\prime}\left\{\Delta\left(z^{\prime}\right)^{\Delta-1} \Phi\left(z^{\prime}, \theta^{\prime}\right)+\left(z^{\prime}\right)^{\Delta} \partial_{z^{\prime}} \Phi\left(z^{\prime}, \theta^{\prime}\right)\right\}+d \theta^{\prime}\left\{\left(z^{\prime}\right)^{\Delta} \partial_{\theta^{\prime}} \Phi\left(z^{\prime}, \theta^{\prime}\right)\right\}\right. \\
& +\left(\mathcal{D}^{\prime} d \theta^{\prime}\right)\left\{2 \Delta\left(z^{\prime}\right)^{\Delta} \Phi\left(z^{\prime}, \theta^{\prime}\right)\right\}+d z^{\prime} d \theta^{\prime}\left\{\left(z^{\prime}\right)^{\Delta} \partial_{z^{\prime}} \partial_{\theta^{\prime}} \Phi\left(z^{\prime}, \theta^{\prime}\right)+\Delta\left(z^{\prime}\right)^{\Delta-1} \partial_{\theta^{\prime}} \Phi\left(z^{\prime}, \theta^{\prime}\right)\right\} \\
& +\left(d z^{\prime}\right)^{2}\left\{\frac{1}{2} \Delta(\Delta-1)\left(z^{\prime}\right)^{\Delta-2} \Phi\left(z^{\prime}, \theta^{\prime}\right)+\frac{1}{2}\left(z^{\prime}\right)^{\Delta} \partial_{z^{\prime}}^{2} \Phi\left(z^{\prime}, \theta^{\prime}\right)+\Delta\left(z^{\prime}\right)^{\Delta-1} \partial_{z^{\prime}} \Phi\left(z^{\prime}, \theta^{\prime}\right)\right\} \\
& +\left(\mathcal{D}^{\prime} d \theta^{\prime}\right)^{2}\left\{\left(z^{\prime}\right)^{\Delta} \Delta(2 \Delta-1) \Phi\left(z^{\prime}, \theta^{\prime}\right)\right\}+\left(\mathcal{D}^{\prime} d \theta^{\prime}\right) d \theta^{\prime}\left\{2 \Delta\left(z^{\prime}\right)^{\Delta} \partial_{\theta^{\prime}} \Phi\left(z^{\prime}, \theta^{\prime}\right)\right\} \\
& \left.+d z^{\prime}\left(\mathcal{D}^{\prime} d \theta^{\prime}\right)\left\{2 \Delta^{2}\left(z^{\prime}\right)^{\Delta-1} \Phi\left(z^{\prime}, \theta^{\prime}\right)+2 \Delta\left(z^{\prime}\right)^{\Delta} \partial_{z^{\prime}} \Phi\left(z^{\prime}, \theta^{\prime}\right)\right\}\right)
\end{aligned}
$$

where we have used that $d\left(\mathcal{D} \theta^{\prime}\right)=\mathcal{D} \theta^{\prime} \mathcal{D}^{\prime}\left(d \theta^{\prime}\right)$ following from (18). Introducing the same expansions as in (32) and (31), and using (22) and (28), one may now compare (30) to (35). We thereby find

$$
\begin{aligned}
z_{i}^{\prime} & =-\sum_{n \in \mathbb{Z}} y_{i, n}\left(z^{\prime}\right)^{n+1}+\sum_{r} \eta_{i, r} \theta^{\prime}\left(z^{\prime}\right)^{r+1} \\
\theta_{i}^{\prime} & =-\frac{1}{2} \sum_{n \in \mathbb{Z}} n y_{i, n} \theta^{\prime}\left(z^{\prime}\right)^{n}-\sum_{r} \eta_{i, r}\left(z^{\prime}\right)^{r}
\end{aligned}
$$

and

$$
\begin{aligned}
& z_{0}^{\prime}=-\sum_{n \in \mathbb{Z}} y_{0, n}\left(z^{\prime}\right)^{n+1}+\sum_{r} \eta_{0, r} \theta^{\prime}\left(z^{\prime}\right)^{r+1}+\frac{1}{2} \sum_{i=1}^{b}\left(z_{i}^{\prime} \partial_{z^{\prime}}+\theta_{i}^{\prime} \partial_{\theta^{\prime}}\right) z_{i}^{\prime} \\
& \theta_{0}^{\prime}=-\frac{1}{2} \sum_{n \in \mathbb{Z}} n y_{0, n} \theta^{\prime}\left(z^{\prime}\right)^{n}-\sum_{r} \eta_{0, r}\left(z^{\prime}\right)^{r}+\frac{1}{2} \sum_{i=1}^{b}\left(z_{i}^{\prime} \partial_{z^{\prime}}+\theta_{i}^{\prime} \partial_{\theta^{\prime}}\right) \theta_{i}^{\prime}
\end{aligned}
$$

As in the case of the conventional superderivative above, $n$ is integer whereas $r$ is integer or half-integer depending on the sector. The expressions (36) and (37) thus represent the solution in the Ramond sector when the sum over $r$ is over $\mathbb{Z}$, while $r \in \mathbb{Z}+\frac{1}{2}$ gives the solution in the NS sector. We wish to point out that, despite the use of a different superderivative and a different definition of a primary field, there is a striking similarity of these solutions with (33) and (34). However, the new results (36) and (37) represent the Ramond sector without the need of introducing a square root. Square roots appear in the NS sector instead. This now gives an alternative way of linking stochastic evolutions in superspace with SCFT. 


\subsection{Expectation values and singular vectors}

Here we wish to indicate how time evolutions of expectation values may be evaluated. In this regard, observables of the process $\mathcal{G}_{t}$ are thought of as functions of $\mathcal{G}_{t}$. The results in [7] on the NS sector, generalizing one of the main results in 4] on ordinary SLE, may be extended straightforwardly to cover the Ramond sector as well. We thus introduce the graded vector fields

$$
\begin{aligned}
\left(\nabla_{n} F\right)\left(\mathcal{G}_{t}\right) & =\left.\frac{d}{d u} F\left(\mathcal{G}_{t} e^{u L_{n}}\right)\right|_{u=0} \\
\left(\mathcal{E}_{r} F\right)\left(\mathcal{G}_{t}\right) & =\left.\frac{d}{d \nu} F\left(\mathcal{G}_{t} e^{\nu G_{r}}\right)\right|_{\nu=0}
\end{aligned}
$$

associated to the generators $L_{n}$ and $G_{r}$. Here $\mathcal{E}_{r}$ and $\nu$ (and of course $G_{r}$ ) are odd, and $F$ is a function admitting a 'sufficiently convergent' Laurent expansion. As always, the Ramond sector corresponds to $r$ being integer, while half-integer $r$ corresponds to the NS sector. Referring to the notation in (31), we find that

$$
\partial_{t} \mathbf{E}\left[F\left(\mathcal{G}_{t}\right)\right]=\mathbf{E}\left[\left(\alpha_{0}(\nabla, \mathcal{E})+\frac{1}{2} \sum_{i=1}^{b} \beta_{i}^{2}(\nabla, \mathcal{E})\right) F\left(\mathcal{G}_{t}\right)\right]
$$

where

$$
\begin{aligned}
\alpha_{0}(\nabla, \mathcal{E}) & =\sum_{n \in \mathbb{Z}} y_{0, n} \nabla_{n}+\sum_{r} \eta_{0, r} \mathcal{E}_{r} \\
\beta_{i}(\nabla, \mathcal{E}) & =\sum_{n \in \mathbb{Z}} y_{i, n} \nabla_{n}+\sum_{r} \eta_{i, r} \mathcal{E}_{r}
\end{aligned}
$$

This follows from (27), (29), $\alpha=\alpha_{0}+\frac{1}{2} \sum_{i=1}^{b} \beta_{i}^{2}$ and

$$
\begin{aligned}
\nabla_{n}\left(\mathcal{G}_{t}^{-N}\right) & =\left.\frac{d}{d u}\left(\left(e^{-u L_{n}} \mathcal{G}_{t}^{-1}\right)^{N}\right)\right|_{u=0} \\
\mathcal{E}_{r}\left(\mathcal{G}_{t}^{-N}\right) & =\left.\frac{d}{d \nu}\left(\left(e^{-\nu G_{r}} \mathcal{G}_{t}^{-1}\right)^{N}\right)\right|_{\nu=0}
\end{aligned}
$$

This may be used to obtain a more direct relationship along the lines of section 2, thereby linking the representation theory of the superconformal algebra, through the construction of singular vectors, to entities conserved in mean under the stochastic process. In particular, it is seen that (39) reduces to

$$
\partial_{t} \mathbf{E}\left[\mathcal{G}_{t}|\Delta\rangle\right]=\mathbf{E}\left[\mathcal{G}_{t}\left(\alpha_{0}+\frac{1}{2} \sum_{i=1}^{b} \beta_{i}^{2}\right)|\Delta\rangle\right]
$$

when $F\left(\mathcal{G}_{t}\right)=\mathcal{G}_{t}|\Delta\rangle$. We should thus look for processes allowing us to put

$$
\left(\alpha_{0}+\frac{1}{2} \sum_{i=1}^{b} \beta_{i}^{2}\right)|\Delta\rangle \simeq 0
$$


in the representation theory. $\mathcal{G}_{t}|\Delta\rangle$ is then a so-called martingale of the stochastic process $\mathcal{G}_{t}$. That is, we should look for processes where (43) corresponds to a null vector in the highest-weight module generated from the vector $|\Delta\rangle$.

\subsubsection{Results for conventional superderivative $D$}

It is discussed in [7] how the NS singular vector (23) may be obtained by considering

$$
\alpha_{0}=-y \eta G_{-3 / 2}, \quad \beta=\sqrt{\kappa}\left(y L_{-1}+\eta G_{-1 / 2}\right), \quad y^{2}=0
$$

This corresponds to

$$
c_{\kappa}=\frac{15}{2}-3\left(\kappa+\frac{1}{\kappa}\right), \quad \Delta_{\kappa}=\frac{2-\kappa}{2 \kappa}
$$

and is in accordance with the ordinary labeling of reducible highest-weight modules of the superconformal algebra with $\Delta=h_{3,1}$. The associated stochastic differential equations are found in [7] to be

$$
\begin{array}{rlrl}
d z^{\prime} & =\frac{y \theta^{\prime} \eta}{z^{\prime}} d t-\left(y+\theta^{\prime} \eta\right) \sqrt{\kappa} d B_{t}, & & \left.z^{\prime}\right|_{t=0}=z \\
d \theta^{\prime} & =\frac{y \eta}{z^{\prime}} d t-\eta \sqrt{\kappa} d B_{t}, & \left.\theta^{\prime}\right|_{t=0}=\theta
\end{array}
$$

with solution

$$
\begin{aligned}
z_{t}^{\prime} & =z+\frac{\theta y \eta}{z} t-(y+\theta \eta) \sqrt{\kappa} B_{t} \\
\theta_{t}^{\prime} & =\theta+\frac{y \eta}{z} t-\eta \sqrt{\kappa} B_{t}
\end{aligned}
$$

Using $D z^{\prime}=\theta^{\prime} D \theta^{\prime}$, it is easily seen to be a superconformal map.

A different scenario based on a two-dimensional Brownian motion is also discussed in [7. Here we shall confine ourselves to one-dimensional Brownian motion only, and our goal now is to examine the stochastic differential equations associated to the level-one singular vector (25) in the Ramond sector. It may be constructed based on

$$
\alpha_{0}=-\frac{1}{2} \epsilon \eta L_{-1}, \quad \beta=\sqrt{\kappa}\left(\epsilon G_{-1}+\eta G_{0}\right)
$$

as one then has

$$
\left(\alpha_{0}+\frac{1}{2} \beta^{2}\right)|\Delta\rangle=\epsilon \eta\left(\left(\kappa-\frac{1}{2}\right) L_{-1}-\kappa G_{-1} G_{0}\right)|\Delta\rangle
$$

This corresponds to

$$
c_{\kappa}=\frac{15}{2}-3\left(\kappa+\frac{1}{\kappa}\right), \quad \Delta_{\kappa}=\frac{6 \kappa-3}{16}
$$


and is in accordance with the ordinary labeling of reducible highest-weight modules of the superconformal algebra with $\Delta=h_{1,2}$. The associated stochastic differential equations are found to be

$$
\begin{aligned}
d z^{\prime} & =\frac{1}{2} \epsilon \eta d t+\sqrt{\kappa}\left(\epsilon\left(z^{\prime}\right)^{-\frac{1}{2}}+\eta\left(z^{\prime}\right)^{\frac{1}{2}}\right) \theta^{\prime} d B_{t}, & & \left.z^{\prime}\right|_{t=0}=z \\
d \theta^{\prime} & =\frac{\kappa \epsilon \eta \theta^{\prime}}{2 z^{\prime}} d t-\sqrt{\kappa}\left(\epsilon\left(z^{\prime}\right)^{-\frac{1}{2}}+\eta\left(z^{\prime}\right)^{\frac{1}{2}}\right) d B_{t}, & & \left.\theta^{\prime}\right|_{t=0}=\theta
\end{aligned}
$$

Substituting

$$
w^{\prime}=z^{\prime}-\sqrt{\kappa}\left(\epsilon\left(z^{\prime}\right)^{-\frac{1}{2}}+\eta\left(z^{\prime}\right)^{\frac{1}{2}}\right) \theta^{\prime} B_{t}
$$

it can be shown that

$$
d w^{\prime}=\frac{1}{2} \epsilon \eta d t,\left.\quad w^{\prime}\right|_{t=0}=z
$$

which can be readily solved:

$$
w^{\prime}=z+\frac{1}{2} \epsilon \eta t
$$

Making the additional substitution

$$
\chi^{\prime}=\theta^{\prime}+\sqrt{\kappa}\left(\epsilon\left(z^{\prime}\right)^{-\frac{1}{2}}+\eta\left(z^{\prime}\right)^{\frac{1}{2}}\right) B_{t}
$$

we have

$$
d \chi^{\prime}=-\frac{\kappa \epsilon \eta \theta^{\prime}}{2 z^{\prime}} d t-\frac{\kappa \epsilon \eta \theta^{\prime} B_{t}}{z^{\prime}} d B_{t},\left.\quad \chi^{\prime}\right|_{t=0}=\theta
$$

with solution

$$
\chi^{\prime}=\theta-\frac{\kappa \epsilon \eta \theta^{\prime} B_{t}^{2}}{2 z^{\prime}}
$$

The original stochastic differential equations (51) are finally found to be solved by

$$
\begin{aligned}
& z^{\prime}=z+\frac{1}{2} \epsilon \eta t+\sqrt{\kappa}\left(\epsilon z^{-\frac{1}{2}}+\eta z^{\frac{1}{2}}\right) \theta B_{t} \\
& \theta^{\prime}=\theta-\sqrt{\kappa}\left(\epsilon z^{-\frac{1}{2}}+\eta z^{\frac{1}{2}}\right) B_{t}+\frac{\kappa \epsilon \eta \theta}{2 z} B_{t}^{2}
\end{aligned}
$$

This is easily seen to correspond to a superconformal map. It is observed that, unlike the NS solution (47), this Ramond solution involves the Brownian motion squared. It is also noted that the solution represents stochastic evolutions in only the 'soul' part of the even co-ordinate, as the complex part of it remains unchanged. As already announced, square roots of $z$ are apparent in these expressions. 


\subsubsection{Results for alternative superderivative $\mathcal{D}$}

We now turn to the alternative superderivative $\mathcal{D}$ using (36) and (37). First we consider the Ramond sector based on the level-one singular vector (25) and the random walk given by (48) and (49). The associated stochastic differential equations read

$$
\begin{array}{rlrl}
d z^{\prime} & =\frac{1}{2} \epsilon \eta d t+\sqrt{\kappa}\left(\eta z^{\prime}+\epsilon\right) \theta^{\prime} d B_{t}, & & \left.z^{\prime}\right|_{t=0}=z \\
d \theta^{\prime} & =\left(\kappa-\frac{1}{2}\right) \frac{\epsilon \eta \theta^{\prime}}{2 z^{\prime}} d t-\sqrt{\kappa}\left(\eta+\frac{\epsilon}{z^{\prime}}\right) d B_{t}, & \left.\theta^{\prime}\right|_{t=0}=\theta
\end{array}
$$

which are seen to be free of square roots of $z^{\prime}$, as expected. These equations can be solved in a manner similar to the one employed above. First we substitute

$$
w^{\prime}=z^{\prime}-\sqrt{\kappa}\left(\eta z^{\prime}+\epsilon\right) \theta^{\prime} B_{t}
$$

yielding the stochastic equation

$$
d w^{\prime}=\frac{1}{2} \epsilon \eta d t,\left.\quad w^{\prime}\right|_{t=0}=z
$$

with solution

$$
w^{\prime}=z+\frac{1}{2} \epsilon \eta t
$$

Making the further substitution

$$
\chi^{\prime}=\theta^{\prime}+\sqrt{\kappa}\left(\eta+\frac{\epsilon}{z^{\prime}}\right) B_{t}+\frac{\kappa \epsilon \eta \theta^{\prime}}{2 z^{\prime}} B_{t}^{2}
$$

yields the stochastic equation

$$
d \chi^{\prime}=-\frac{\epsilon \eta \theta^{\prime}}{4 z^{\prime}} d t,\left.\quad \chi^{\prime}\right|_{t=0}=\theta
$$

with solution

$$
\chi^{\prime}=\theta-\frac{\epsilon \eta \theta^{\prime} t}{4 z^{\prime}}
$$

The solution to the original set of equations (59) finally reads

$$
\begin{aligned}
z^{\prime} & =z+\frac{1}{2} \epsilon \eta t+\sqrt{\kappa}(\eta z+\epsilon) \theta B_{t} \\
\theta^{\prime} & =\theta-\frac{\epsilon \eta \theta}{4 z} t-\sqrt{\kappa}\left(\eta+\frac{\epsilon}{z}\right) B_{t}+\frac{\kappa \epsilon \eta \theta}{2 z} B_{t}^{2}
\end{aligned}
$$

This can be substituted directly into (17) to check that it corresponds to a superconformal map. Two different, stochastically evolving systems, (58) and (66), have thus been found to correspond to the same singular vector (25). Only one of them contains square roots of $z$. 
To 'complete the picture', we now examine the stochastic differential equations following from considering the NS singular vector (23) in the realm of the alternative superconformal structure based on $\mathcal{D}$. Following our general prescription, we find

$$
\begin{array}{rlrl}
d z^{\prime} & =-y \eta \theta^{\prime}\left(z^{\prime}\right)^{-\frac{1}{2}} d t+\sqrt{\kappa}\left(\eta \theta^{\prime}\left(z^{\prime}\right)^{\frac{1}{2}}-y\right) d B_{t}, & \left.z^{\prime}\right|_{t=0}=z \\
d \theta^{\prime}=\left(1-\frac{\kappa}{2}\right) y \eta\left(z^{\prime}\right)^{-\frac{3}{2}} d t+\sqrt{\kappa}\left(\frac{1}{2} y \theta^{\prime}\left(z^{\prime}\right)^{-1}-\eta\left(z^{\prime}\right)^{-\frac{1}{2}}\right) d B_{t}, & \left.\theta^{\prime}\right|_{t=0}=\theta
\end{array}
$$

The new co-ordinate

$$
w^{\prime}=z^{\prime}+\sqrt{\kappa}\left(y-\eta \theta^{\prime}\left(z^{\prime}\right)^{\frac{1}{2}}\right) B_{t}
$$

then satisfies the stochastic differential equation

$$
d w^{\prime}=-y \eta \theta^{\prime}\left(z^{\prime}\right)^{-\frac{1}{2}} d t,\left.\quad w^{\prime}\right|_{t=0}=z
$$

with solution

$$
w^{\prime}=z-y \eta \theta^{\prime}\left(z^{\prime}\right)^{-\frac{1}{2}} t
$$

Likewise, the substitution

$$
\chi^{\prime}=\theta^{\prime}+\sqrt{\kappa}\left(\eta\left(z^{\prime}\right)^{-\frac{1}{2}}-\frac{1}{2} y \theta^{\prime}\left(z^{\prime}\right)^{-1}\right) B_{t},\left.\quad \chi^{\prime}\right|_{t=0}=\theta
$$

must respect

$$
d \chi^{\prime}=\left(1+\frac{\kappa}{2}\right) y \eta\left(z^{\prime}\right)^{-\frac{3}{2}} d t+\kappa y \eta\left(z^{\prime}\right)^{-\frac{3}{2}} B_{t} d B_{t}
$$

with solution

$$
\chi^{\prime}=\theta+y \eta\left(z^{\prime}\right)^{-\frac{3}{2}} t+\frac{1}{2} \kappa y \eta\left(z^{\prime}\right)^{-\frac{3}{2}} B_{t}^{2}
$$

One could have made a further substitution and considered the equation

$$
d\left(\left(w^{\prime}\right)^{\frac{3}{2}} \tilde{\chi}^{\prime}\right)=d\left(\left(w^{\prime}\right)^{\frac{3}{2}}\left\{\chi^{\prime}-\frac{\kappa}{2} y \eta\left(z^{\prime}\right)^{-\frac{3}{2}} B_{t}^{2}\right\}\right)=y \eta d t,\left.\quad\left(w^{\prime}\right)^{\frac{3}{2}} \tilde{\chi}^{\prime}\right|_{t=0}=z^{\frac{3}{2}} \theta
$$

which is solved trivially and without reference to the original equations (67). In either case, one may conclude that the original equations are solved by

$$
\begin{aligned}
& z^{\prime}=z-y \eta \theta z^{-\frac{1}{2}} t+\sqrt{\kappa}\left(\eta \theta z^{\frac{1}{2}}-y\right) B_{t} \\
& \theta^{\prime}=\theta+y \eta z^{-\frac{3}{2}} t+\sqrt{\kappa}\left(\frac{1}{2} y \theta z^{-1}-\eta z^{-\frac{1}{2}}\right) B_{t}-\frac{1}{2} \kappa y \eta z^{-\frac{3}{2}} B_{t}^{2}
\end{aligned}
$$

This is seen to correspond to a superconformal map. 


\section{Conclusion}

The results of [7] on the links between stochastic evolutions of superconformal maps and the NS sector of $N=1$ SCFT have been extended to the Ramond sector, as well as to an unconventional superconformal structure underlying $N=1$ superspace and $N=1$ SCFT. In the usual treatment of the Ramond sector, it is found that square roots of the even co-ordinate, $z$, have to be introduced. Based on the alternative superconformal structure and using the associated definition of a primary field found in [10, a modified set of equations are found. They give results for the Ramond sector without square roots, while square roots appear in the NS sector. To the best of our knowledge, this is the first time that such a primary field has been used, and results compared to the more conventional treatment of the Ramond and NS sectors.

\section{Acknowledgements}

JN is funded by PPARC and thanks them for their financial support. The authors thank the EUCLID network, contract HPRN-CT-2002-00325, for funding the first EUCLID network conference (Firenze, 2003) where the present collaboration was conceived.

\section{References}

[1] O. Schramm, Israel J. Math. 118 (2000) 221.

[2] G.F. Lawler, O. Schramm, W. Werner, Acta Math. 187 (2001) 237; Acta Math. 187 (2001) 275; Ann. Inst. Henri Poincaré PR 38 (2002) 109; Conformal restriction: the cordal case, math.PR/0209343.

[3] S. Rohde, O. Schramm, Basic properties of SLE, math.PR/0106036.

[4] M. Bauer, D. Bernard, Phys. Lett. B 543 (2002) 135; Commun. Math. Phys. 239 (2003) 493.

[5] R. Friederich, W. Werner, Conformal restriction, highest-weight representations and SLE, math-ph/0301018.

[6] F. Lesage, J. Rasmussen, SLE-type growth processes and the Yang-Lee singularity, to appear in J. Math. Phys., math-ph/0307058.

[7] J. Rasmussen, Stochastic evolutions in superspace and superconformal field theory, to appear in Lett. Math. Phys., math-ph/0312010.

[8] A. Rogers, Supersymmetry and Brownian motion on supermanifolds, quant-ph/0201006. 
[9] V.G. Kac, J.W. van de Leur, On classification of superconformal algebras, in Strings '88, eds. S.J. Gates, C.R. Preitschopf, W. Siegel (World Scientific, 1989) p.77-106.

[10] J. Nagi, J. Math. Phys. 45 (2004) 2492.

[11] M. Dörrzapf, Rev. Math. Phys. 11 (1999) 137. 